\title{
Lymphatische Malformationen
}

Gefäßanomalien werden gemäss der ISSVA (International Society for the Study of Vascular Anomalies) -Klassifikation seit 1982 in Tumoren und Malformationen unterteilt. Diese Unterteilung basiert auf dem biologischen und klinischen Verhalten dieser Anomalien. Im Laufe der Zeit wurde diese Klassifikation weiter verfeinert und weitere Gefäßerkrankungen mit unterschiedlicher Komplexität wurden hinzugefügt. Vaskuläre Malformationen werden weiter unterteilt in: Einfache Malformationen, in Kombination mit anderen Malformationen, vaskuläre Malformationen benannt nach großen Gefäßen sowie in Kombination mit anderen Gefäßanomalien. Die einfachen Gefäßmalformationen können zudem in „Low Flow“und „High Flow“-Malformationen unterteilt werden. Die korrekte Verwendung dieser Klassifikation ist essenziell für das Management dieser Patienten. Entsprechend hat der Radiologe in der Diagnostik der Gefäßanomalien eine wichtige Rolle und beeinflusst die weitere Therapie entscheidend.

Die hier besprochenen lymphatischen Malformationen sind kongenitale, gutartige Erkrankungen der Lymphgefäße. Sie gehören zur Gruppe der „Low Flow“-vaskulären Malformationen und imponieren klinisch als weiche, nicht pulsierende Masse mit einer normalen, darüber liegenden Haut. Eine lymphatische Malformation besteht aus malformierten lymphatischen Gefäßen, welche aufgrund eines Defekts während der embryonalen Lymphangiogenese entstehen. Mehrere Gene wurden beschrieben, welche die Entwicklung der Lymphgefäße beeinflussen. Die dilatierten Gänge oder Zysten sind mit einer proteinreichen Flüssigkeit gefüllt und haben keine Verbindung zum normalen Lymphsystem.
Normalerweise sind die lymphatischen Malformationen entweder unmittelbar nach der Geburt erkennbar oder werden in den ersten 2 Lebensjahren manifest. $48 \%$ kommen im Bereich Kopf und Hals vor, $42 \%$ am Torso und $10 \%$ intrathorakal oder intraabdominal.

Lymphatische Malformationen können weiter in makrozystische und mikrozystische Läsionen unterteilt werden. Die Unterscheidung bleibt in der Literatur etwas vage. Sinn macht wohl die Unterteilung, ob Zysten im Ultraschall erkennbar sind (Makrozysten) oder eben lediglich eine „solide“ Läsion abgrenzbar ist (Mikrozysten). Diese Einteilung hat letztendlich eine Konsequenz bezüglich Therapie sowie auch auf das langfristige Ergebnis.

Lymphatische Malformationen kommen solitär oder multifokal vor und wachsen generell langsam mit dem Patienten eine spontane Involution kommt selten vor. In einigen Fällen wachsen lymphatische Malformationen sehr rasch und komprimieren je nach Lokalisation wichtige Strukturen, wie z. B. Luftwege oder den Augennerv im Falle eines orbitalen Befalls. Meistens sind Haut und Muskel von der Malformation betroffen, selten auch Knochen, der Gastrointestinaltrakt oder die Lungen. Intraläsionale Blutungen oder Infektionen können bei lymphatischen Malformationen akut auftreten, welche zu einer massiven Schwellung beitragen können oder aber auch zu einem hämodynamisch relevanten Hämoglobin-Abfall.

Die Bildmodalität der Wahl für zystische lymphatische Malformationen sind die Sonografie und die Magnetresonanztomografie (MRT), welche gewöhnlich vor Therapiebeginn durchgeführt werden. Die MRT stellt die Gesamtausdehnung der Malformation besser dar, insbesondere bei sehr tiefen, großen und komplexen Läsionen. Die Sonografie hingegen gibt sehr wichtige Hinweise bezüglich Strategie der Therapie. Zystische lymphatische Malformationen sind hypointens in $\mathrm{T} 1$ und hyperintens in T2 (aufgrund des hohen Flüssigkeitsgehalts). Makrozysten zeigen ein Enhancement der Septen, mikrozystische lymphatische Malformationen hingegen zeigen häufig ein nur flaues, inhomogenes Enhancement. Flüssigkeit-Spiegel entstehen aufgrund Einblutungen in die Zysten. Eine Kontrast-Lymphangiografie (z. B. intranodale MR-Lymphangiografie) ist für zystische lymphatische Malformationen nicht notwendig. Hingegen ist die intranodale Kontrast-Lymphangiografie bei bestimmten lymphatischen Malformationen mit abnormalen Lymphbahnen und Lymphlecks des Ductus thoracicus und bei Patienten mit chylösem Aszites oder Chylothorax sehr hilfreich.

Wiederkehrende Schwellungen, Schmerzen, Infektionen, funktionelle Einschränkungen und Einblutungen sind Indikationen für die Behandlung von zystischen lymphatischen Malformationen. Insbesondere auch bei Kindern im Schulalter ist die Ästhetik ein wichtiger Grund, eine lymphatische Malformation zu behandeln. Trotz der hohen Rate von Rezidiven (25 - 52\%) und Komplikationen (12,5 - 44\%) ist die chirurgische Exzision historisch gesehen die Therapie der Wahl der lymphatischen Malformationen. Eine wesentlich weniger invasive Therapieform ist die bildgesteuerte Sklerotherapie sowie die medikamentöse Therapie mit Rapamycin (hier nicht diskutiert). Der Vorteil der minimalinvasiven Sklerothe- 
rapie beinhaltet ein geringeres Risiko von Nerven- oder Gefäßschädigungen insbesondere an anatomisch schwierigen Lokalisationen (wie Hals), eine raschere Erholung nach dem Eingriff und insbesondere auch keine Hinterlassung von chirurgischen Narben. Bei makrozystischen lymphatischen Malformationen kann die Sklerotherapie kurativ sein, und mindestens $90 \%$ der Patienten zeigen eine signifikante Verbesserung der Ästhetik sowie der Symptome. Mikrozystische lymphatische Malformationen hingegen sind mittels Sklerotherapie schwieriger zu behandeln, aber auch da bietet die minimalinvasive Therapie eine gewisse Größenreduktion der Läsion.

Als Sklerosierungsmittel kommt hauptsächlich Sodium Tetradecyl Sulfat (STS), Doxycyclin, Bleomycin und Ethanol zur Anwendung oder eine Kombination derselbigen. Bei größeren Zysten (> $5 \mathrm{~cm}$ ) können Drains eingelegt werden, um die Sklerotherapie am Folgetag zu wiederholen. Die Wahl und Dosis des Sklerosierungsmittels hängt ab von der anatomischen Lokalisation der lymphatischen Malformation sowie vom Volumen der Zysten. Nach der Therapie ist eine Schwellung der Läsion zu erwarten,
Schmerzen sind eher unüblich. Gelegentlich können Einblutungen vorkommen, Hautblasen sind selten. Bei größeren Zysten ist eine Wiederholung der Therapie nach ca. 2 Monaten zu empfehlen.

Die intranodale MR-Lymphangiografie (MRL) zur dynamischen Darstellung der Lymphgefäße hat in den letzten Jahren erneut zunehmend an Bedeutung gewonnen. Arbeiten von Itkin et al. haben gezeigt, dass die MRL die Prognose eines Patienten mit einem kongenitalen oder postoperativen Chylothorax voraussagen kann. Dazu wird inguinal jeweils ein Lymphknoten mittels einer Nadel angestochen und fixiert. Danach wird der Patient in das MRT transferiert und es werden initial native Sequenzen akquiriert. Danach wird das Kontrastmittel (Gadolinium, $1: 2$ verdünnt) langsam über die Lymphknoten gespritzt (max. $0,5 \mathrm{ml} / \mathrm{min}$ ) und es werden zusätzliche, dynamische Sequenzen akquiriert. Gewisse Patienten können dann von einer Embolisation des Ductus thoracicus profitieren. Der Zugang dazu erfolgt in der Regel über die Cysterna chyli antegrad oder retrograd über die V. sub- clavia oder als Direktpunktion des Ductus thoracicus.

Interessenkonflikt

Die Autoren geben an, dass kein Interessenkonflikt besteht.

Autorinnen/Autoren

Ralph Gnannt

Universitäts-Kinderspital Zürich

Korrespondenzadresse

Ralph Gnannt

Universitäts-Kinderspital Zürich

Ralph.Gnannt@kispi.uzh.ch

Bibliografie

DOI https://doi.org/10.1055/a-0943-1098

Online-Publikation: 2019

Fortschr Röntgenstr 2019; 191: S111-S112

(c) Georg Thieme Verlag KG, Stuttgart · New York ISSN 1433-5972 